\title{
Desenvolvimento Evolutivo do Ambiente Virtual de Ajuda para Suporte Pedagógico e Operacional de Sistema Tutor Inteligente de Apoio Educacional
}

\author{
Vinícius da Silva Carvalho, Gesiel dos Santos Freitas, Natanael Natons de \\ Oliveira Fernandes, Vandor Roberto Vilardi Rissoli \\ Engenharia de Software - Universidade de Brasília \\ Faculdade do Gama (FGA) - Gama Leste, Brasília - DF \\ vinisilvacar@aluno.unb.br, vandorissoliegmail.com
}

\begin{abstract}
The improvement of web interaction support environment for users of Intelligent Tutor System SAE, has become crucial due to increased use and popularization of this educational software. Creating, editing and updating of virtual static pages in the help system enhances the description and view the step by step processes required to achieve the objectives sought by users. The use and adaptation of sequential instructions, combining textual and visual communication, and the use of interface similar to the SAE system, contribute to the improvement in the effectiveness of the help system and provides safe operation of your various user profiles.
\end{abstract}

Resumo. A melhoria de interação web ao ambiente de suporte aos usuários do Sistema Tutor Inteligente SAE, tornou-se fundamental devido a maior utilização e popularização desse software educacional. A criação, edição e atualização de páginas estáticas virtuais no sistema de ajuda aprimora a descrição e visualização de passo a passo dos processos necessários para atingir os objetivos almejados pelos usuários. $O$ uso e adaptação de instruções sequenciais, unindo comunicação textual e visual, além do uso de interface semelhante ao sistema SAE, contribuem para o avanço na efetividade do sistema de ajuda e fornece segurança na operação de seus diferentes perfis de usuários.

\section{Introdução}

A evolução das tecnologias digitais alcançou diversas áreas, entre elas, a educação, agregando novos recursos educacionais com intuito de torná-la mais eficiente e coerente com a realidade atual. Entre esses recursos, foi destacado neste trabalho o SAE (Sistema de Apoio Educacional), que apoia o processo de ensino e aprendizagem, de forma personalizada, de seus usuários estudantes (RISSOLI, 2007). O SAE é um Sistema Tutor Inteligente (STI) que fornece um ambiente de interação para os diferentes perfis de indivíduos envolvidos diretamente no processo educacional, além de propiciar acesso e assistência a outros perfis de usuário que participam na administração e gerência desse processo pedagógico. 
O projeto SAE é originário de pesquisas envolvendo professores e estudantes de três instituições brasileiras de ensino superior - Universidade Católica de Brasília (UCB), Universidade Federal do Rio Grande do Sul (UFRGS) e Pontifícia Universidade Católica do Rio Grande do Sul (PUC-RS). Ele objetiva empregar recursos tecnológicos adequados a uma metodologia educacional coerente ao ensino-aprendizagem almejado, consistindo num projeto multidisciplinar envolvendo, principalmente, as áreas de Educação, Psicologia e Informática (RISSOLI, 2007).

O SAE utiliza como principal recurso de suporte tecnológico um tipo de software educacional que possibilita interação adequada, por meio da rede mundial de computadores (Internet), podendo ser empregado como sistema de apoio ao ensinoaprendizagem praticado à distância ou presencialmente, bem com a combinação destas duas formas de instrução (semipresencial).

A elaboração deste software, é baseada nas concepções fundamentais da Teoria da Aprendizagem Significativa, proposta por David Paul Ausubel (1968), e colaborado com a extensão da arquitetura tradicional dos Sistemas Tutores Inteligentes para um Assistente Virtual de Ensino Inteligente (AVEI), viabilizou a elaboração do Sistema de Suporte Educacional SAE.

Dentre vários recursos desse software, o Sistema Tutor Inteligente (STI) tem sido empregado - com sucesso - em diversas disciplinas dos cursos de bacharelado em Ciência da Computação da UCB e em Engenharia de Software da Universidade de Brasília (UnB), além da iniciação de seu uso pela Secretaria de Estado de Educação do Distrito Federal (SEEDF).

Este sistema vem sendo ampliado com novos módulos que almejam assistir e apoiar os principais envolvidos neste processo de formação educacional, sendo necessária a elaboração de um material instrutivo (manual eletrônico) sobre o uso correto deste sistema como ferramenta de suporte ao ensino-aprendizagem personalizado às características individuais de seus estudantes.

Esse manual instrutivo do sistema SAE é composto por páginas virtuais estáticas que objetivam apresentar, na forma "passo a passo", como proceder no uso mais operacional dos recursos disponíveis nesse STI. Esse manual ou guia possui como padrão apresentar as instruções de forma textual e ilustrativa, oferecendo interface semelhante ao sistema em estudo para melhor adequação e entendimento de seu funcionamento, assim como de sua interface de interação.

Para a disseminação e melhoria no processo de interação com os usuários, o projeto SAE dispõe do ambiente conhecido como AjudaSAE, vinculado ao SAE e que tem a finalidade de orientar os seus usuários mediante o perfil de cada indivíduo deste, permitindo uma interação adequada e coerente aos diferentes objetivos destes perfis no STI (SILVA, 2010).

A Figura 1 mostra a página virtual inicial do AjudaSAE. 


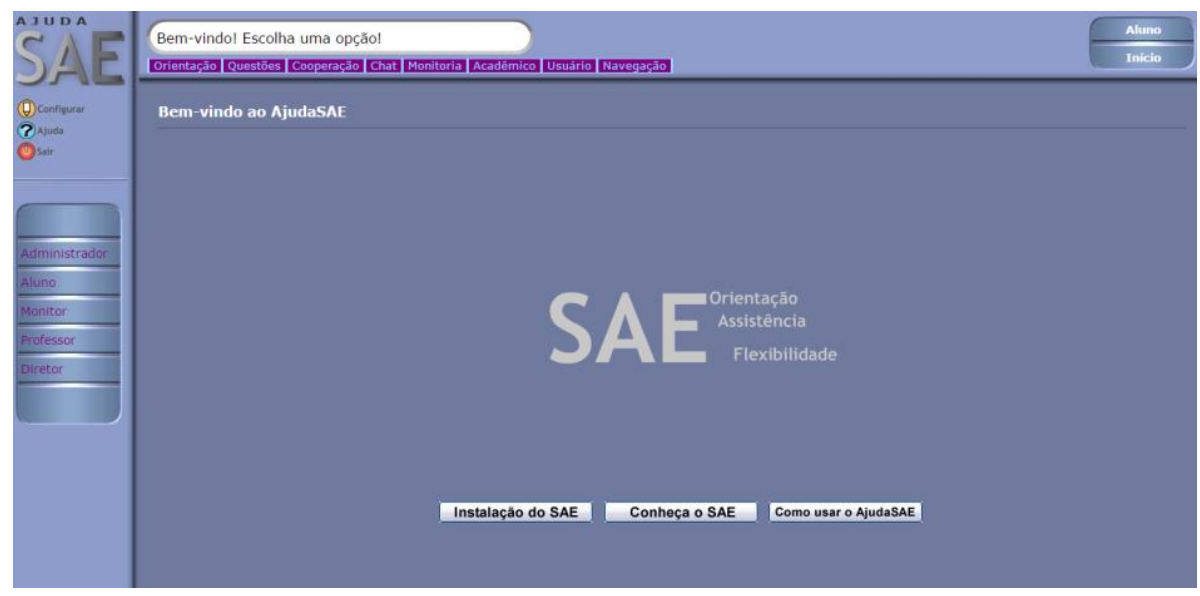

Figura 1. Janela inicial e interativa do manual eletrônico AjudaSAE.

\section{Metodologia}

Para Rosenbaum, Kantner e Dworman (2005), a ajuda é importante quando é organizada na interface do usuário, tornando-a uma memória descritiva ou disponível apenas a alguns cliques de distância. Estes autores apresentam diferentes tipos de conteúdo de ajuda: Processual (informações que descrevem a forma de realizar uma tarefa), Interface (instruções sobre a interface e sobre como utilizar cada um de seus elementos), Domínio de informação (informação sobre os recursos do software), Suplementar (informação sobre termos técnicos, como em um glossário), e Metainstruções (informações sobre o uso do software).

Já a autora Ascencio (2000), classifica os tipos de conteúdo de ajuda da seguinte forma: Orientada ao objetivo (demonstrando o que é possível fazer com o software); Descritiva (mencionando o que é e qual a utilidade de componentes do programa); Procedimento (apresentando como é realizada tal tarefa); Interpretativa (expondo o porquê e como acontece um cálculo ou tarefa no programa); e Navegação (que objetiva deixar o usuário ciente de sua localização no software). Essa classificação foi estendida por Herrmann e Silveira (2005), que incluíram a ajuda de Exemplo (sugerindo a apresentação de exemplos sobre como usar o Sistema).

A elaboração desse trabalho envolveu a otimização de dois tipos de conteúdo essenciais ao material de apoio existente no ambiente de ajuda do SAE, denominado AjudaSAE. São eles: Navegação (possibilidades de rápida navegação no ambiente para auxílio coerente ao perfil de cada usuário), e Procedimento (o conteúdo de auxílio que esclarece como é realizada uma funcionalidade específica no sistema) (PELISSARI, 2010).

De acordo com Oliveira e Silveira (2007), em uma pesquisa realizada com usuários de software educacional (alunos, professores e coordenadores de laboratório de informática), notou-se que esses usuários possuem perfis diferentes e por isso necessitam de informações diferenciadas. Mas, apesar da análise de diversificação de perfis de usuários (não só de perfil computacional e de conhecimento, mas, também, de objetivos diferentes no uso do software), as informações encontradas nos sistemas de ajuda geralmente são apresentadas de uma única forma, sem se preocupar com estas diferenças. 
O Manual Eletrônico do sistema SAE obedece essa diferença, assim como o próprio sistema em estudo que possui a interface semelhante ao sistema de apoio educacional a fim de familiarizar o usuário com os estilos de tela e modo de navegação do sistema. Entretanto, apenas no sistema de ajuda é possível definir qual perfil de usuário é necessário para apresentação pariforme ao que o usuário em questão possui acesso, por meio do menu lateral. Logo após, o usuário poderá selecionar qual esfera (módulo) deseja consultar, por meio do menu superior disponível tanto no manual AjudaSAE, quanto no sistema SAE.

Observe na Figura 2 a primeira imagem capturada do SAE e a segunda disponível no AjudaSAE ilustrando a navegação que deve ser realizada pelo usuário com o efeito do sombreamento, destacando, na segunda imagem, a navegação correta indicada.
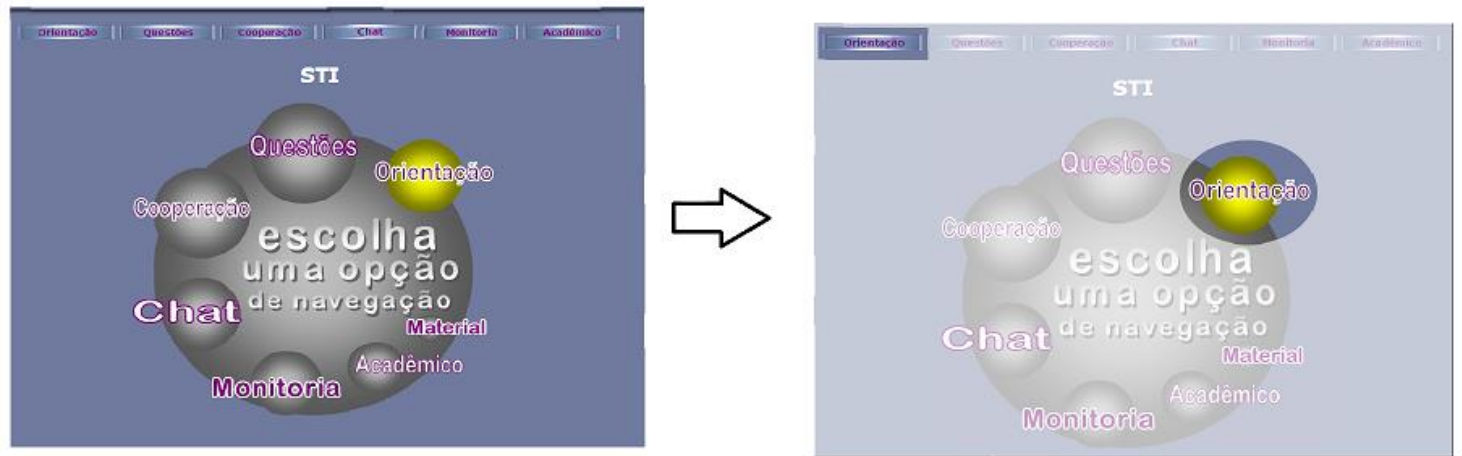

Figura 2. Demonstração do trabalho de disposição ilustrativa para correta navegação no Sistema SAE.

Mediante contato com usuários (alunos, monitores, professores, diretores e administradores) do sistema de ajuda foi observada a necessidade de maior acessibilidade ao manual, além de melhoria na leitura das instruções disponibilizadas, explorando a rapidez e fácil interpretação do conteúdo apresentado aos usuários. Com isso, as atividades presentes nesse trabalho incluem pesquisa e desenvolvimento de conteúdo com maior aptidão a leitura dinâmica do manual eletrônico, que se dá, principalmente, pelas imagens das janelas capturadas do próprio SAE, tornando possível as ilustrações significativas às instruções "passo a passo" para realização da atividade desejada.

Com a maior distribuição do sistema pela rede educacional, e observada a prática habitual em sua utilização, o AjudaSAE pôde ser elaborado para a leitura de seu conteúdo ser mais rápida e fácil aos seus usuários, a fim de permitir que eles consigam realizar as instruções fornecidas de forma mais adequada aos seus interesses. Neste caso, considera-se a importância da rapidez na leitura, interpretação e reprodução (ou realização) das instruções indicadas pelo AjudaSAE.

A Figura 3 ilustra a evolução na elaboração cuidadosa dos conteúdos que podem ser mais rápidos à compreensão dos usuários do $\mathrm{SAE}$ que precisam de auxílio ágil enquanto o utilizam.

As páginas virtuais do AjudaSAE não são criadas por especialistas que possuem estudo adequado para a construção desse conteúdo, são os seus próprios implementadores de funcionalidades no SAE. E como o AjudaSAE é composto por páginas virtuais de texto criados por vários autores, observa-se descrições de forma 
V Congresso Brasileiro de Informática na Educação (CBIE 2016)

Anais dos Workshops do V Congresso Brasileiro de Informática na Educação (CBIE 2016)

irregular e em desacordo com a ortografia brasileira, o que, em alguns casos, dificultavam os usuários.
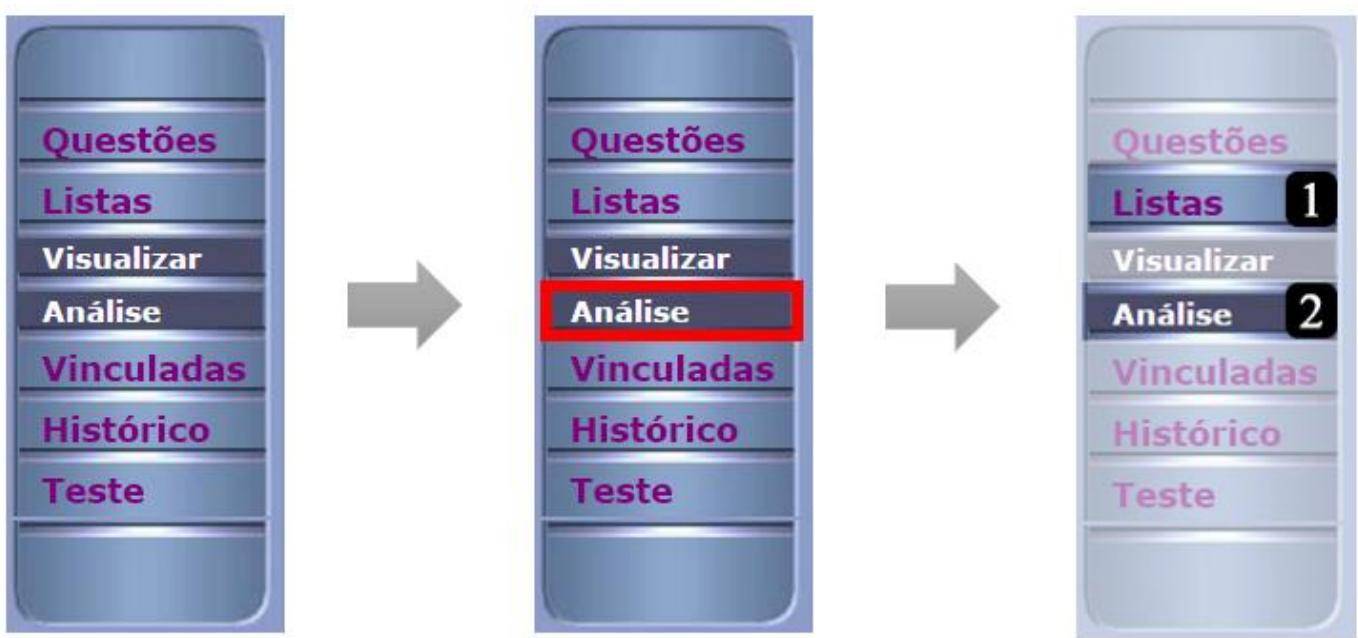

Figura 3. Exemplo da evolução visual de instruções no AjudaSAE.

Alguns textos do AjudaSAE não respeitavam o Acordo Ortográfico da Língua Portuguesa, assinado em Lisboa, em 1990, além de ter alguns problemas de concordância resultantes de conteúdos novos que eram integrados aos já existentes.

A Figura 4 mostra parte de uma página virtual do AjudaSAE.

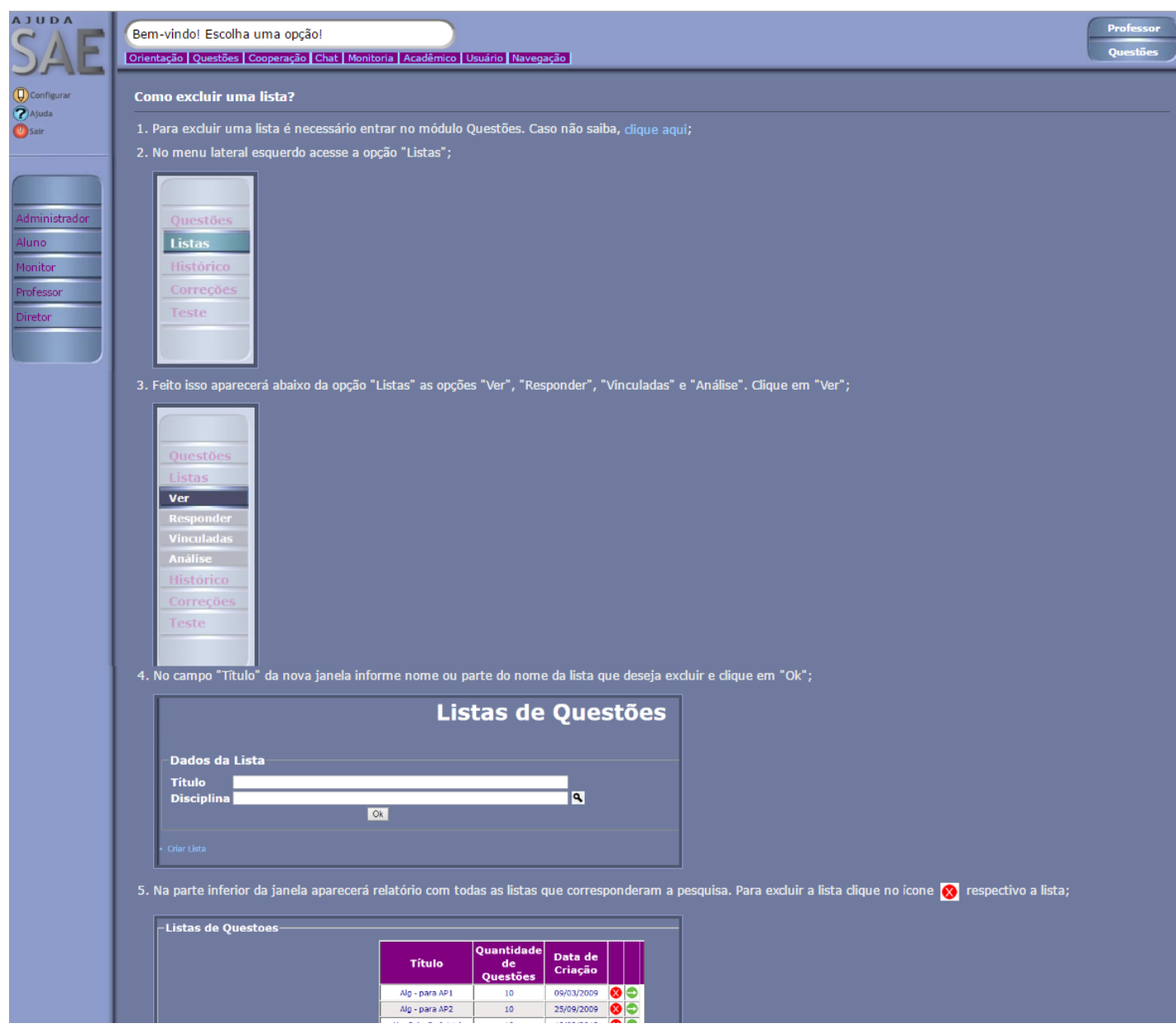


V Congresso Brasileiro de Informática na Educação (CBIE 2016)

Anais dos Workshops do V Congresso Brasileiro de Informática na Educação (CBIE 2016)

\section{Figura 4. Apresentação instrutiva do AjudaSAE para excluir uma lista de exercícios do sistema SAE.}

$\mathrm{Na}$ Figura 4 é possível observar como as imagens estão presentes no manual eletrônico do SAE, sendo demonstrado o progresso na melhoria da visualização ágil fornecida ao usuário na forma "passo a passo" para que ele atinja seu objetivo, operando o SAE corretamente.

As tecnologias utilizadas para a realização desse trabalho envolveram um editor de código fonte multiplataforma chamado Sublime Text, o editor de imagens bitmap e desenho vetorial Adobe Fireworks CS6, o software de captura de tela da Microsoft Snipping Tool, além de controladores de versão $S V N$ e Git. O gerenciador de repositórios de software utilizado foi o GitLab.

O resultado desse trabalho foi elaborado por meio de páginas virtuais, desenvolvidas em HTML (HyperText Markup Language), respeitando o padrão visual definido no SAE em CSS (Cascading Style Sheet) específicos e reaproveitáveis, de modo a facilitar a interação do usuário na operação do sistema de ajuda e do próprio software educacional SAE (MARCONDES, 2005).

Esse trabalho teve como foco principal o aperfeiçoamento do conteúdo de ajuda proveniente de versões anteriores do AjudaSAE, no qual foram realizadas identificações de falhas, pesquisas e correções em seus conteúdos; simplificação de instruções; aprimoramento do conteúdo; seguimento na evolução no ambiente de ajuda e no sistema SAE; manutenção e realização de testes, de forma a disponibilizar o ambiente de produção do AjudaSAE de maneira segura.

\section{Resultados}

O manual eletrônico do SAE (AjudaSAE) e o sistema SAE se assemelham visualmente com a finalidade de facilitar a interação entre eles, compartilhando a interface definida coerentemente no Sistema. Essa abordagem visa auxiliar seus usuários nos recursos interativos durante a navegação no ambiente de ajuda de maneira similar ao uso do próprio sistema.

A partir das atividades, realizaram-se a criação e manutenção de páginas HTML do AjudaSAE, que estão hospedadas no sítio virtual cae.ucb.br/ajudasae. Foram atualizados os comandos descritos de acordo com a versão atual do sistema, a adaptação de páginas para apresentação padrão de conteúdo, edição e atualização de imagens, em que se tornou necessária devido a evolução do próprio $\mathrm{SAE}$, o apontamento de operações por imagens, a fim de ajudar o usuário de forma rápida e eficaz, fornecendo uma nova capacidade de auxílio ágil aos usuários do sistema e buscando o sucesso operacional de todos os envolvidos (principalmente os discentes e docentes).

O trabalho realizado nesse período contribuiu para expansão e melhoria do conteúdo de ajuda referente a sistema $\mathrm{SAE}$, nos cinco diferentes perfis e onze módulos presentes no sistema. A partir da Tabela 1, é possível aferir a criação de novos conteúdos e, consequentemente, o maior material de apoio a ser disponibilizado.

A criação de novos conteúdos de ajuda foram realizadas mediante solicitações dos usuários do sistema, com o contato direto ou por correio eletrônico do grupo SAE 
V Congresso Brasileiro de Informática na Educação (CBIE 2016)

Anais dos Workshops do V Congresso Brasileiro de Informática na Educação (CBIE 2016)

(sae.contato@gmail.com). Foi observado o aumento de 36,2\% no total de arquivos do ambiente AjudaSAE, o que equivale a 590 arquivos criados durante esse período.

Tabela 1. Relação da quantidade de arquivos antes e após o trabalho realizado.

\begin{tabular}{|c|l|l|l|}
\hline \multicolumn{1}{|c|}{ Módulos } & \multicolumn{1}{|c|}{$\begin{array}{c}\text { Quantidade de } \\
\text { Arquivos anteriores }\end{array}$} & $\begin{array}{c}\text { Quantidade de } \\
\text { Arquivos após o } \\
\text { trabalho }\end{array}$ \\
\hline 1 & Módulo Acadêmico & 179 arquivos & 387 arquivos \\
\hline 2 & Módulo Chat & 42 arquivos & 70 arquivos \\
\hline 3 & Módulo Conheça SAE & 76 arquivos & 77 arquivos \\
\hline 4 & Módulo Cooperação & 133 arquivos & 211 arquivos \\
\hline 5 & Módulo Instalação & 150 arquivos & 150 arquivos \\
\hline 6 & Módulo Monitoria & 116 arquivos & 147 arquivos \\
\hline 7 & Módulo Navegação & 75 arquivos & 69 arquivos \\
\hline 8 & Módulo Orientação & 271 arquivos & 336 arquivos \\
\hline 9 & Módulo Questões & 464 arquivos & 602 arquivos \\
\hline 10 & Módulo Solicitação & 18 arquivos & 18 arquivos \\
\hline 11 & Módulo Usuário & 106 arquivos & 153 arquivos \\
\hline
\end{tabular}

Comparando a criação de novas páginas virtuais com instruções e orientações aos usuários do SAE por módulos tem-se a análise comparativa mostrada na Figura 5.

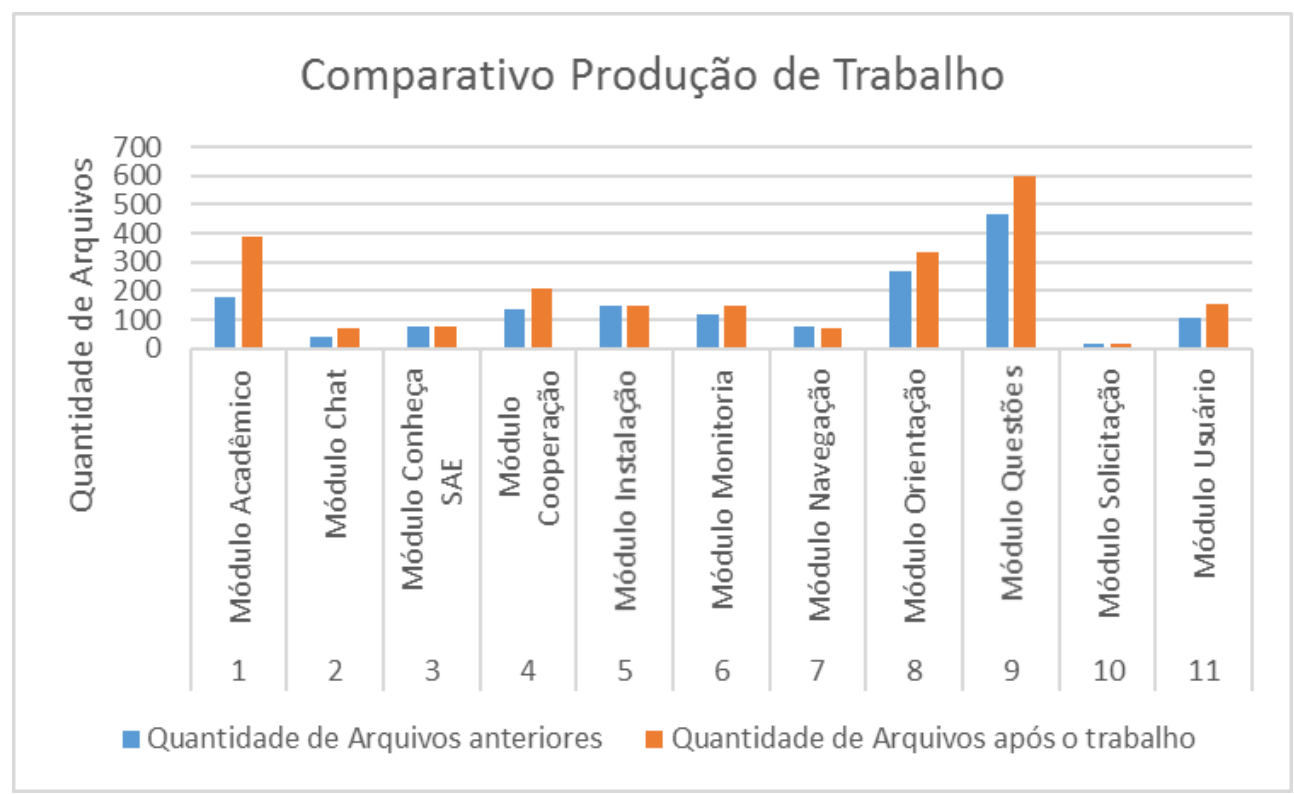


V Congresso Brasileiro de Informática na Educação (CBIE 2016)

Anais dos Workshops do V Congresso Brasileiro de Informática na Educação (CBIE 2016)

\section{Figura 5. llustração comparativa da produção de páginas do AjudaSAE pelos módulos do SAE existentes no momento.}

Além das novas páginas criadas no sistema de ajuda, a correção de conteúdos existentes consistiu na tarefa mais trabalhosa realizada durante o período de atividades desse trabalho, muitos erros gramaticais e ortográficos foram ajustados, imagens foram redefinidas e instruções reformuladas, adotando um padrão entre as páginas.

\section{Conclusão}

O trabalho supracitado foi realizado com satisfatoriamente. Foi notada maior facilidade dos usuários em relação a leitura dos conteúdos do ambiente de ajuda do SAE devido a maior objetividade e simplificação das instruções com as imagens disponibilizadas para cada pergunta condizente com as principais dúvidas apuradas. Erros ortográficos foram corrigidos, junto com a evolução do conteúdo explicativo, de modo a deixar as instruções mais claras e fáceis de serem compreendidas com a utilização de princípios básicos de interação humano-computador (ROGERS, 2013).

Dessa forma, o ambiente AjudaSAE tem promovido agilidade ao acesso e melhor utilização do Sistema Tutor Inteligente SAE, diminuindo o esforço cognitivo de seus usuários, que podem manter o foco no conteúdo letivo da aprendizagem e nas atividades sugeridas por este software educacional, a fim de propiciar um processo de assimilação mais significativo a estrutura cognitiva de cada aprendiz usuário do SAE e do AjudaSAE.

\section{Referências}

Ascencio, A. F. G. (2000) Método Heurístico para Projeto de Interfaces Inteligentes com Usabilidade (Dissertação de Mestrado), Universidade Federal do Rio Grande do Sul.

Ausubel, D. P.; Novak, J. D. e Hanesian, H. (1968) Educational Psychology: A cognitive view. New York: Holt, Rinehart and Winston.

Herrmann, M. e Silveira, M. S. (2005) "Sistemas de Ajuda Online em Softwares Educacionais: algumas considerações e percepções". In: Anais do XVI Simpósio Brasileiro de Informática na Educação, p. 477-485.

Oliveira, M. R. e Silveira, M. S. (2007) "Algumas Considerações sobre a Construção do Conteúdo de Sistemas de Ajuda Online para Software Educacional”. In: Anais do XVIII Simpósio Brasileiro de Informática na Educação, p.223-226.

Marcondes, C. A. (2005) HTML 4.0 Fundamental: A base para programação para Web. Érica.

Pellissari, E. e Silveira, M. S. (2010). Estruturação e Apresentação de Conteúdos de Sistemas de Ajuda On-line para Softwares Educacionais. In: Anais do Simpósio Brasileiro de Informática na Educação.

Rissoli, V. R. V. (2007) "Uma proposta metodológica de acompanhamento personalizado para aprendizagem significativa apoiada por um assistente virtual de ensino inteligente", Tese (Doutorado) - Universidade Federal do Rio Grande do Sul. Programa de Pós-Graduação em Informática na Educação, Porto Alegre BR-RS. 
V Congresso Brasileiro de Informática na Educação (CBIE 2016)

Anais dos Workshops do V Congresso Brasileiro de Informática na Educação (CBIE 2016)

Rogers, Y.; Sharp, H. e Preece, J. (2013), Design de interação: além da interação humano-computador. $3^{\mathrm{a}}$ ed. Porto Alegre: Bookman, 2013.600p.

Rosenbaum, S.; Kantner, L. e Dworman, G. (2005) "Helping Users to Use Help: Results from Two International Conference Workshops". In: Proceedings of IEEE International Professional Communication Conference, p.181-187.

Silva, G.; Girotto, V. e Rissoli, V. (2010), Ambiente de ajuda do Sistema Tutor Inteligente. Departamento de Ciência da Computação da Universidade Católica de Brasília. 